\title{
On Laplace Transforms Near the Origin
}

\author{
By R. Wong*
}

Abstract. Let $f(t)$ be locally integrable on $[0, \infty)$ and let $L\{f\}(s)$ denote the Laplace transform of $f(t)$. In this note, we prove that if $f(t) \sim t^{-\beta} \Sigma_{n=0}^{\infty} a_{n}(\log t)^{-n}$ as $t \rightarrow \infty$, where $0 \leqslant \operatorname{Re} \beta<1$, then $L\{f\}(s) \sim s^{\beta-1} \Sigma_{n=0}^{\infty} c_{n}(\log 1 / s)^{-n}$ as $s \rightarrow 0$ in $|\arg s| \leqslant \pi / 2$ $-\Delta$, the $c_{n}$ being constants.

1. Introduction. Let $f(t)$ be locally integrable on $[0, \infty)$ and let $L\{f\}$ denote the Laplace transform of $f(t)$. That is,

$$
L\{f\}=\int_{0}^{\infty} f(t) e^{-s t} d t,
$$

whenever the integral on the right converges. In a recent paper, Handelsman and Lew [2] have studied the asymptotic behavior of $L\{f\}$ as $s \rightarrow 0$, when $f(t)$ satisfies

$$
f(t) \sim \exp \left(-c t^{p}\right) \sum_{m, n=0}^{\infty} c_{m n} t^{r}(\log t)^{n} \quad \text { as } t \rightarrow \infty,
$$

where $p>0, \operatorname{Re} c \geqslant 0, \operatorname{Re} r_{m} \downarrow-\infty$ as $m \rightarrow \infty$, and the set $\left\{n: c_{m n} \neq 0\right\}$ is finite for each $m$. In this note, we consider the case

$$
f(t) \sim t^{-\beta} \sum_{n=0}^{\infty} a_{n}(\log t)^{-n} \quad \text { as } t \rightarrow \infty,
$$

where $0 \leqslant \operatorname{Re} \beta<1$. Our result will complement that of Handelsman and Lew.

2. Main Theorem. For convenience, we introduce the notation

$$
L_{c}\{f\}=\int_{c}^{\infty} f(t) e^{-s t} d t
$$

where $c$ is a fixed real number $>1$. In [3], it was shown that for any complex number $\beta$ with $\operatorname{Re} \beta<1$,

$$
L_{c}\left\{t^{-\beta}(\log t)^{-n}\right\} \sim s^{\beta-1}\left(\log \frac{1}{s}\right)^{-n} \sum_{r=0}^{\infty}\left(\begin{array}{c}
-n \\
r
\end{array}\right) \Gamma^{(r)}(1-\beta)\left(\log \frac{1}{s}\right)^{-r}
$$

as $s \rightarrow 0$ in $S(\Delta)$, where

Received July 23, 1973.

AMS (MOS) subject classifications (1970). Primary $41 \mathrm{~A} 60$.

Key words and phrases. Laplace transform, asymptotic expansion, Ramanujan function.

* Research partially supported by the National Research Council of Canada under Contract No. A7359.

Copyright (c) 1975. American Mathematical Society 


$$
S(\Delta)=\{s:|\arg s| \leqslant \pi / 2-\Delta\}
$$

Let

$$
c_{n}=\sum_{r=0}^{n} a_{n-r}\left(\begin{array}{c}
r-n \\
r
\end{array}\right) \Gamma^{(r)}(1-\beta) .
$$

We are now ready to state and prove our main result.

THEOREM. If $f(t)$ is locally integrable on $[0, \infty)$ and satisfies $(1.3)$, then as $s \rightarrow 0$ in $S(\Delta)$

$$
L\{f\} \sim s^{\beta-1} \sum_{n=0}^{\infty} c_{n}\left(\log \frac{1}{s}\right)^{-n},
$$

where the coefficients $c_{n}$ are given in (2.4).

Proof. For any $c>1$,

$$
L\{f\}=L_{c}\{f\}+\int_{0}^{c} f(t) e^{-s t} d t=L_{c}\{f\}+O(1)
$$

as $s \rightarrow 0$ in $S(\Delta)$.

Writing

$$
f(t)=\sum_{n=0}^{N} a_{n} t^{-\beta}(\log t)^{-n}+R_{N}(t)
$$

gives

$$
L_{c}\{f\}=\sum_{n=0}^{N} a_{n} L_{c}\left\{t^{-\beta}(\log t)^{-n}\right\}+r_{N}
$$

where

$$
r_{N}=\int_{c}^{\infty} R_{N}(t) e^{-s t} d t
$$

From (1.3), it follows that there are constants $K>0$ and $c>1$ such that

$$
\left|R_{N}(t)\right| \leqslant K\left|t^{-\beta}(\log t)^{-N-1}\right| \text { for } t \geqslant c .
$$

Hence, by (2.2),

$$
\begin{aligned}
\left|r_{N}\right| & \leqslant K \int_{c}^{\infty} t^{-\gamma}(\log t)^{-N-1} e^{-\sigma t} d t \\
& =O\left(\sigma^{\gamma-1}(\log \sigma)^{-N-1}\right) \quad \text { as } \sigma \rightarrow 0
\end{aligned}
$$

where $\gamma=\operatorname{Re} \beta$ and $\sigma=\operatorname{Re} s$. Since $|s| \sin \Delta \leqslant \sigma \leqslant|s|$ for any $s \in S(\Delta),(2.11)$ is equivalent to

$$
r_{N}=O\left(|s|^{\gamma-1}(\log |s|)^{-N-1}\right)=O\left(s^{\beta-1}(\log s)^{-N-1}\right)
$$

as $s \rightarrow 0$ in $S(\Delta)$. Coupling the results (2.8) and (2.12), we obtain

$$
L_{c}\{f\}=\sum_{n=0}^{N} a_{n} L_{c}\left\{t^{-\beta}(\log t)^{-n}\right\}+O\left(s^{\beta-1}(\log s)^{-N-1}\right)
$$


as $s \rightarrow 0$ in $S(\Delta)$. Since the $O$-term in (2.6) may be included in that of (2.12), (2.13) implies

$$
L\{f\}=\sum_{n=0}^{N} a_{n} L_{c}\left\{t^{-\beta}(\log t)^{-n}\right\}+O\left(s^{\beta-1}(\log s)^{-N-1}\right)
$$

as $s \rightarrow 0$ in $S(\Delta)$. In view of (2.2), each term in (2.14) can be expanded in powers of $(\log 1 / s)^{-1}$. Hence, by regrouping the terms, we have for any $N \geqslant 0$

$$
L\{f\}=s^{\beta-1}\left[\sum_{n=0}^{N} c_{n}\left(\log \frac{1}{s}\right)^{-n}+O\left((\log s)^{-N-1}\right)\right]
$$

as $s \rightarrow 0$ in $S(\Delta)$. This completes the proof of our theorem.

3. An Example. The Ramanujan function is defined by

$$
N(s)=\int_{0}^{\infty} \frac{e^{-s t}}{t\left\{\pi^{2}+(\log t)^{2}\right\}} d t .
$$

Recently, Bouwkamp [1] obtained the asymptotic expansion

$$
N(s) \sim \sum_{n=0}^{\infty} c_{n}(\log s)^{-n-1} \quad \text { as } s \rightarrow \infty,
$$

where the coefficients were determined by the generating function

$$
\frac{1}{\Gamma(1-x)}=\sum_{n=0}^{\infty} c_{n} \frac{x^{n}}{n !} \text {. }
$$

Our aim is to find the asymptotic behavior of $N(s)$ as $s \rightarrow 0$.

Integrating by parts, we obtain from (3.1)

$$
N(s)=\frac{1}{2}+\frac{s}{\pi} \int_{0}^{\infty} \tan ^{-1}\left(\frac{1}{\pi} \log t\right) e^{-s t} d t .
$$

The function $\tan ^{-1}(\log t / \pi)$ has the convergent expansion

$$
\tan ^{-1}\left(\frac{1}{\pi} \log t\right)=\frac{\pi}{2}-\sum_{n=0}^{\infty} \frac{(-1)^{n}}{2 n+1}\left(\frac{\pi}{\log t}\right)^{2 n+1}
$$

for $t>e^{\pi}$. Hence, the conditions of the theorem are trivially satisfied and we have

$$
N(s) \sim 1-\sum_{\nu=0}^{\infty} a_{\nu}\left(\log \frac{1}{s}\right)^{-\nu-1}
$$

as $s \rightarrow 0$ in $S(\Delta)$, where

$$
a_{\nu}=\sum_{2 n+r=\nu} \frac{(-1)^{n} \pi^{2 n}}{2 n+1}\left(\begin{array}{c}
-2 n-1 \\
r
\end{array}\right) \Gamma^{(r)}(1) .
$$

It is interesting to note that these coefficients are precisely the ones given by Bouwkamp for the asymptotic expansion of $N(s)$ as $s \rightarrow \infty$. To see this, we recall the identity 
$\Gamma(x) \Gamma(1-x)=\pi / \sin \pi x$. Hence, from (3.3),

$$
c_{\nu}=\nu ! \sum_{2 n+r=\nu} \frac{(-1)^{n} \pi^{2 n}}{(2 n+1) !} \frac{\Gamma^{(r)}(1)}{r !} .
$$

Comparing (3.7) and (3.8), we have

$$
a_{\nu}=(-1)^{\nu} c_{\nu}, \quad \nu=0,1,2, \cdots .
$$

Department of Mathematics

University of Manitoba

Winnipeg, Manitoba, Canada

1. C. J. BOUWKAMP, "Note on an asymptotic expansion," Indiana Univ. Math. J., v. 21, 1971, pp. 547-549.

2. RICHARD A. HANDELSMAN \& JOHN S. LEW, “Asymptotic expansion of Laplace transforms near the origin," SIAM J. Math. Anal., v. 1, 1970, pp. 118-129. MR 41 \#4142.

3. R. WONG, "On a Laplace integral involving logarithms," SIAM J. Math. Anal., v. 1, 1970, pp. 360-364. MR 43 \#7842. 